\title{
El imaginario estético en la comproducción virtual de ecofábulas
}

\section{Tukip Likapaykaa Ishkapwayuchikaaćhu Wirtuwal Ecobabulakunaćhu \\ Ora kenganijintagantsi okantgetanaka samgenare kantëgotsatagansipage}

Recepción: 19 agosto 2020 Corregido: 16 diciembre 2020 Aprobación: 01 marzo 2021

Waldemar José Cerrón Rojas

Nacionalidad: peruana / Universidad Nacional del Centro del Perú Correo: wjcerron@uncp.edu.pe. /ORCID: https://orcid.org/oooo-0002-5664-1769

Bertha Rojas López Nacionalidad:peruana / Investigadora Independiente / Correo: rumi45@hotmail.com

\section{Resumen}

En el presente artículo se visibiliza la trascendencia del imaginario estético como soporte de la comproducción virtual de ecofábulas. EI imaginario estético constituye la fuente creativa de la cual emergen las formas figurativas para la creación literaria, en este caso, la comproducción virtual de ecofábulas. La comproducción virtual de ecofábulas constituye una conexión intertextual entre una fábula (producción precedente) con otra que necesariamente emerge al momento de comprender lo que se lee, actividades que se realizan en espacios virtuales. La investigación fue de tipo aplicada de nivel experimental con diseño pre experimental. Se aplicó el método de la modelación y el histórico lógico. Los instrumentos utilizados fueron la ficha de observación del imaginario estético y la ficha de análisis de contenido de la comproducción virtual de ecofábulas. El grupo de estudio estuvo conformado por 35 estudiantes de la Carrera Profesional de Lenguas, Literatura y Comunicación. Se concluye que el nivel de eficacia del imaginario estético en la comproducción de ecofábulas es alto, como lo evidencia la publicación de las ecofábulas comproducidas y la estimación del p-valor: $0,00=0 \%$ con un nivel de significancia de $\alpha=0,05$. De esta manera, se abandona la tradición de que lo leído, queda en lo comprendido; sino más bien la obligatoriedad de materializar el acto productivo subyacente en toda comprensión de lectura.
Palabras clave:

Imaginario, comproducción virtual, ecofábulas.

\section{Lisichiku limaykuna:}

Likana, wirtuwal ishkayp lulay, ecofabulakuna.

\section{Nibarintsi katingaro:} kenganijintagantsi, kantagantsipage osiagantsitakijenga kantëgosatagantsipage.

\section{Datos de los autores}

Waldemar José Cerrón Rojas es docente e investigador en educación, estética y literatura. Doctor en Ciencias de la educación por la Universidad Enrique Guzmán y Valle La Cantuta. Magister en Didáctica universitaria por la Universidad Nacional del Centro del Perú.

Bertha Rojas López docente e investigadora en educación y literatura. Doctora en Ciencias de la educación por la Universidad Enrique Guzmán y Valle La Cantuta. Magister en Didáctica universitaria por la Universidad Nacional del Centro del Perú. 


\title{
The Aesthetic Imaginary in the Virtual Comproducción of Ecofábulas
}

\begin{abstract}
In this article, the transcendence of the aesthetic imaginary as a support for the comproducción of eco-fables is made visible. The aesthetic imaginary constitutes the creative source from which the figurative forms for literary creation emerge, in this case, the virtual comproducción of eco-fables. The virtual comproducción of eco-fables constitutes an intertextual connection between a fable (previous production) with another that necessarily emerges at the moment of understanding what is read, activities that are carried out in virtual spaces. The research was applied at an experimental level with a pre-experimental design. The method of modeling and logical history were applied. The instruments used were the observation sheet for the aesthetic imaginary and the content analysis sheet for the virtual comproducción of eco-fables. The study group was made up of 35 students from the Professional Career of Languages, Literature and Communication. It concludes that the level of efficacy of the aesthetic imaginary in the comproducción of eco-fables is high, as evidenced by the publication of the eco-fables comproducidas and the estimation of the $p$-value: $0,00=0 \%$ with a significance level of $\alpha=0,05$. In this way, the tradition that what is read remains in what is understood is abandoned; but rather the obligation to materialize the underlying productive act in all reading comprehension.
\end{abstract}

\section{O imaginário estético na comprodução virtual de ecofábulas}

\section{Resumo}

Neste artigo, torna-se visível a transcendência do imaginário estético como suporte da comproduçao virtual de ecofábulas. O imaginário estético constitui a fonte criativa da qual emergem as formas figurativas para a criação literária, neste caso, a comprodução virtual de ecofábulas . A comprodução virtual de ecofábulas constitui uma conexão intertextual entre uma fábula (produção precedente) com outra que necessariamente emerge ao momento de compreender o que se lê, atividades que se realizam em espaços virtuais. A pesquisa foi aplicada em nível experimental com um desenho pré-experimental. Aplicou-se o método de modelagem e o lógico-histórico. Os instrumentos utilizados foram a ficha de observação do imaginário estético e a ficha de análise de conteúdo da comprodução virtual das ecofábulas. O grupo de estudos foi formado por 35 alunos do Curso Profissional de Línguas, Literatura e Comunicação. Conclui-se que o nível de eficácia do imaginário estético na comprodução de ecofábulas é alto, conforme evidenciado pela publicação das ecofábulas comproduzidas e estimativa do $p$-valor: $0,00=0 \%$ com nível de significância de $\alpha=0,05$. Desse modo, abandona a tradição de que o que é lido permanece no que é compreendido; mas, sim, a obrigatoriedade de materializar o ato produtivo subjacente em toda compreensão de leitura.

\section{Keywords}

Imaginary,

virtual

comproducción, eco-fables.
Palavras-chave:

Imaginário, comprodução virtual, ecofábulas. 


\section{Introducción}

La formación de las sensibilidades humanas es una constante del devenir histórico en el incesante ideal por alcanzar niveles elevados de humanización. En este proceso formativo, la Literatura adquiere trascendencia generacional, mediante el efecto estético figurado, el cual dinamiza emociones, sentimientos, pensamientos y conocimientos contenidos en el imaginario del desarrollo social. La comprensión de estas resonancias o giros del lenguaje literario, compromete a los lectores con dos condiciones necesarias; la primera en la transfiguración denotativa a la connotativa y la segunda la producción como respuesta a esta interpretación; ambas constituyen el soporte epistemológico de la comproducción lectora virtual.

El imaginario estético literario configura la posibilidad infinita de la producción literaria, cada sociedad, por generaciones alimenta al imaginario lo creado, recreado en el tiempo y el espacio. El avance de la tecnología, sobre todo en la comunicación electrónica, ofrece la oportunidad de comprender y producir, diversos géneros literarios, entre ellos, las ecofábulas, cuya cualidad expresa la necesidad de la formación y reafirmación de las sensibilidades sociales consideradas dentro del imaginario como bello en contradicción de lo feo. Al respecto Pavón (2016) refiere:

Nuestra época, por ejemplo, está marcada profundamente por la omnipresencia y omnipotencia del capital, la técnica, lo razonable, lo cuantitativo y lo tecnológico, a su vez las sociedades que habitan en este espacio de tiempo han de transformarse consumistas, individualistas, superfluas, efímeras (Castoriadis), líquidas (Bauman) o de espectáculo (Debord).

El acto comproductivo interconecta las percepciones, interpretaciones, vivencias, experiencias y conocimientos e ideales del escritor y del lector mediante sus imaginarios. Concordante con (Maccioni, 2016) a pesar de la existencia de diferencias de carácter estético entre las producciones literarias, se fabula el reinvento territorial, los ríos, islas, que al momento de escribirlo traslucen su origen imaginario, del cual emergen la unidad de la tierra del agua y la palabra que los nombran, así como los sujetos, leyes, regularidades sociales en que las habitan.

En esa misma dirección Ariste, E.; Zapana, E. (2011); Moscoso, E. (2015) Cerrón W.; Rojas, B. (2014), (2016), (2018) investigaron sobre la ecofábula y sus repercusiones en desarrollo de las habilidades comunicativas, corporales, aplicación del método wanka para la creación de fábulas; asi como el análisis del efecto estético de las ecofábulas, las bases epistemológicas de la comproducción lectora virtual. Estas investigaciones visibilizan la posibilidad interconectiva de la revitalización dialéctica de los significados estéticos.

El objetivo principal de la investigación fue es establecer el nivel de eficacia de la comproducción lectora virtual de ecofábulas en estudiantes. El escenario investigativo se desarrolló con estudiantes de la Universidad Nacional del Centro del Perú. Mediante la observación estructurada se detectó que la mayoría presentaban dificultades presentaban dificultades en la comproducción de ecofábulas porque no conectaban el imaginario estético literario como soporte fundamental y necesario para la comproducción de ecofábulas. La conectividad interactiva entre el imaginario estético literario transparenta la comproducción de ecofábulas virtuales favorable a la conciencia ecológica de las nuevas generaciones.

Durante la comproducción virtual de ecofábulas los estudiantes elegían temas que no estaban vinculados a temas ecológicos. Se evidenciaron incoherencia entre la situación inicial, inicio del conflicto, conflicto, resolución del conflicto, por lo que la situación final se tornaba 
difusa y un grupo consideraba que no era posible su realización porque no interrelacionaban, ni activaban el imaginario estético durante el proceso comproductivo. El espacio virtual fue de gran ayuda para observar estas dificultades y superarlas en tiempo sincrónico y asincrónico, puestos que pudieron intercambiar la teoría y la práctica comproductiva.

La comproducción virtual de ecofábulas es un acto creativo y recreativo del imaginario eco fabulesco soportado en la transfiguración de su forma y contenido utilizando plataformas virtuales. El inicio del acto recreativo está marcado por la existencia de una ecofábula correspondiente al imaginario de escritor, la cual motiva, guía y revitaliza su contenido mediante la expresión de actitudes humanas favorables o desfavorables hacia la naturaleza; mientras que la forma se presenta a través de personajes, diálogos, performance, contemporizados e interconectados con imaginario del comproductor en los espacios virtuales.

El imaginario estético literario soporta la comproducción virtual de las ecofábulas porque constituye la fuente, el manantial, el espacio donde se "aloja" la sinergia de las sensibilidades sociales, imagen literaria y categorías estéticas. El efecto estético de la imagen verbal transfigura la sensibilidad de conocimientos, filosofía, educación, política e ideología, la interacción simbólica de la imagen de lo típico individual, racional emocional, objetivo subjetivo figurados en las categorías de lo bello feo, trágico cómico, heroico, medroso, sublime, vil, humano, frívolo; sutil o grotesco. En el imaginario se revitalizan constantemente la necesidad de proyectar mejores condiciones de vida espiritual, frente al avance de la ciencia y la tecnología. A respecto Neuburger, (2020) manifiesta que:

Los estudios en torno a la autonomía del arte constituyen en nuestros días un amplio y prolífico campo de discusión, en la medida, en que cada época se ha ocupado de discutir los alcances de aquello que en términos de especificidad/inespecificidad otorga sentido a las prácticas estéticas. Quizá nuestro tiempo hace propia dicha discusión en un escenario estético, político, filosófico que desde hace varias décadas se piensa, al menos, en términos de agotamiento. La clausura, en tanto diagnóstico de época, se infiltró no sólo en el campo de la filosofía o la historia, sino, aún más, en lo que refiere a la producción artística. (p.43)

En la búsqueda de nuestras identidades y formas culturales de interactuar con las personas, recurrimos a los imaginarios, los que ineludiblemente contienen valores éticos, estéticos y morales porque son los parámetros con los cuales se resuelven problemas sociales, sobre todo la proyección de las actitudes y comportamiento de los hombres a decir de (Hernández Moredo, 2020, p. 59). "En la indagación sobre nuestras raíces, atender a esta esfera reviste significancia no solo científica sino social, dada la importancia de la dimensión estética tanto en la vida cotidiana como en el modo de afrontar la crisis de valores en el mundo contemporáneo".

La comproducción virtual de ecofábulas transfigura imágenes, figuras, símbolos e interaccionismo simbólico de la diversidad de meta espacios ecológicos y culturales. Conecta la relación adversa o armónica de las acciones del hombre con los elementos emergentes del contexto natural y teologizado, en el cual desarrolla su vida cotidiana, muchas veces sin percatarse de sus efectos identitarios o alienantes. Todo comproductor trae consigo un imaginario ecológico que deviene de sus vivencias, experiencias y expectativas, el que en la actualidad puede ser almacenado y revitalizado con el uso de diversas plataformas.

Las sociedades modernas híper tecnologizadas y mediatizadas están expuestas constantemente a una inducción, refuerzo y desacreditación de diversas cosmovisiones imaginarias 
sociales. Éstas (re)aparecen sin cesar en las variadas plataformas que ofrecen las nuevas tecnologías, por esta razón las sociedades están constantemente azotadas de ciertas estructuras imaginarias que constituyen y mantienen la institución socio-histórica en la que habita el anthropos. (Pavón, 2016).

La comproducción virtual de ecofábulas es el resultado de la unidad de la comprensión y producción soportado en imaginario estético literario relacionadas con la temática ecológica en escenarios virtuales. Los comproductores identifican en la primera etapa (transfase comprensora) el sentido y significado de la propuesta estética de las ecofábulas y luego en la segunda etapa (transfase productora) asignan significados emergentes a imágenes, figuras, símbolos e interaccionismos simbólicos en ecofábulas emergentes.

Las ecofábulas comproducidas (emergentes) cíclicamente generan otras ecofábulas. De manera didáctica síncrona y asíncrona fueron recreándose diversos metas espacios ecológicos como ríos, quebradas, bosques, árboles, animales y diversas actitudes humanas de la ineludible transfiguración e interacción armónica, propias del imaginario socio ambiental económico. En las ecofábulas se transparentan las acciones estéticas bellas o feas de la humanidad a favor o en contra de su verdadero y más grande hogar: La naturaleza.

Los comproductores de ecofábulas incrementaron de manera cooperativa, al imaginario estético literario, propuestas con sentido y significado ecológico social armónico. Interconectaron plataformas virtuales y redes sociales ecológicas del conocimiento, lo cual hizo posible, el intercambio comproductivo de ecofábulas. Al respecto Montoya (2019) "Las interacciones ecológicas, así como las redes que forman estas interacciones, son un componente clave de la biodiversidad de los ecosistemas. Existen al menos tres razones ecológicas fundamentales para incluir interacciones en los programas de restauración" (p.12)

La sociedad en general podrá interactuar con la diversidad de la información ecológica de la comproducción de ecofábulas virtuales. Asumimos como trascendental, el conocimiento y práctica del imaginario estético literario para la comproducción virtual de ecofábulas en el proceso cibertural científico y artístico para contrarrestar las deformidades socio ecológicas, que pretenden destruir naturaleza, en consecuencia, a la misma humanidad. De esta manera las ecofábulas comproducidas están al servicio de la conciencia ecológica positiva de las comunidades locales y globales.

Un ejemplo constituye la ecofábula EL Grillo y la Culebra de las autoras Lisbeth Corpus García y Torres Huaricapcha Karen comproducida de la ecofábula la Mariposa y el Gusano de Bertha Rojas López y Waldemar José Cerrón Rojas.

\section{Ecofábula inicial: La Mariposa y el Gusano}

Sobre el pétalo de una rosa, bailoteaba una mariposa.

De pronto dijo: -- ¡Puffff...!, qué repugnancia los que se arrastran.

Debajo del rosal se encontraba un gusano y éste gritó:

--¡Mariposa! recuerda que tú fuiste, un gusano repugnante.

La mariposa respondió:

- ¡Sí!, fui un gusano, pero jamás me arrastré como aquellos que se arrastran sin ser gusanos.

Moraleja: Alcanzar los ideales con honor y dignidad.

Ecofábula comproducida: El Grillo y la Culebra

https://padlet.com/corpusgarcialis20191/d6oikq6vt4vw 
Sobre el pétalo de un clavel bailoteaba un grillo;

De pronto dijo - Qué vergüenza los que se arrastran

Debajo del clavel estaba una culebra, la que al escuchar respondió:

- Grillo, recuerda que antes tú fuiste pequeño y apenas podías caminar.

El grillo respondió: Sí, yo fui pequeño; pero nunca me arrastré tanto.

\section{Materiales y Métodos}

La aplicación experimental del imaginario estético en la comproducción virtual de ecofábulas se realizó en la Facultad de Educación de la Universidad Nacional del Centro del Perú. Los materiales fueron almacenados, aplicados y transferidos en el meta espacio virtual https://padlet.com/josecin4/ecofavirtual. La muestra estuvo conformada por 28 estudiantes de la Carrera Profesional de Lenguas, Literatura y Comunicación de la nombrada universidad. Investigación cuantitativa, explicativa, en la que se aplicó el método científico, específicamente el experimental con diseño pre experimental $(\mathrm{G}:=01 \mathrm{Xo2})$.

La publicación virtual de ecofábulas comproducidas, estadística descriptiva e inferencial visibilizan los resultados del nivel de eficacia del imaginario estético en la comproducción virtual de ecofábulas. Se diseñó la plataforma virtual www.padlet.com/josecin4/ecofavirtual, la cual permitió la conexión con obras, textos y materiales de trabajo. Se contrastó la hipótesis mediante la estimación del p-valor: $0.00=0 \%$ con un nivel de significancia de $\alpha=0,05$. Los instrumentos empleados fueron la ficha de análisis de contenido del efecto estético y comproducción virtual de ecofábulas.

Tabla 1

Resultados de la Prueba de Entrada y Salida

\begin{tabular}{lcc}
\hline & Prueba de Entrada & Prueba de Salida \\
\hline Media & 3,8 & 17,57 \\
Mediana & 4,00 & 18,00 \\
Moda & 4,00 & 18,00 \\
Desviación estándar & 1,45 & 1,80 \\
Varianza & 2,10 & 3,25 \\
\hline Suma & 136,00 & 615,00 \\
\hline
\end{tabular}

Nota. Las calificaciones promedio de la prueba de salida del imaginario estético en la comproducción virtual de ecofábulas superaron los calificativos de la prueba de entrada luego de la aplicación experimental de dos meses de aplicación.

Se puede observar que en la prueba de salida los estudiantes alcanzan un promedio de 17 , 57; mientras que en la prueba de entrada sólo alcanzaron 3,08. Lo que nos indica que el imaginario estético tiene un alto nivel de eficacia para la producción virtual de ecofábulas.

\section{Discusión de resultados}

La investigación demostró el nivel de eficacia del imaginario estético en la comproducción virtual de ecofábulas en estudiantes de la Facultad de Educación de la Universidad Nacional del Centro del Perú. La base teórica que soportó la investigación fue la comproducción lectora virtual propuesta por Cerrón (2016); por lo que se extrapolaron la identidad pedagógica, 
visibilización del sentido ideológico, trasmutación de limitaciones históricas del imaginario estético ecológico a la situación inicial, inicio del conflicto ecológico, conflicto, resolución del conflicto y situación final de la fábula emergente.

Los resultados que se evidencian con la estimación del p-valor: $0.00=0 \%$ con un nivel de significancia de $\alpha=0,05$, concuerdan con Ariste y Zapana (2011) quienes al aplicar la estrategia de la ecofábula lograron demostrar su eficacia en la comunicaciòn oral, que también es posible en la comunicación escrita y virtual, en este caso la comproducción virtual de ecofábulas. En la misma dirección Cerrón y Rojas (2014) demuestran la eficacia del método wanka para la producción de ecofábulas mediante la estructura de ituación inicial, inicio del conflicto, conflicto, resolución del conflicto y situación final en el formato físico, pertinente también, para la comproducción virtual de ecofàbulas.

En cuanto a la importancia de limaginario estético para la comproducción de ecofábulas, coincidimos con lo afirmado por Cerrón (2014), quien destaca el valor e importancia de las categorías estéticas enmarcadas en lo bello y lo feo, típico e individual, cómico, trágico, heroicas o cómicas provenientes del imaginario estético de manera general y particular de los comproductores. Así mismo coincidimos con Montes y Moscoso (2015), quien remarca la posibilidad de crear ecofábulas combinando el imaginario matemático, estético, ecológico de manera integral, vivencial, analítica y reflexiva, aspectos necesarios para la comproducción virtual de ecofábulas.

Desde las bases teóricas para la comproducción de ecofábulas, concordamos con la investigación desarrollada por Cerrón (2016) en la que se reafirma la importancia de las formas de conocer y hacer conocer la realidad (bases epistemologicas de la comproducción lectora virtual) considerando la imagen, el símbolo, interaccionismo simbólico de las limitaciones históricas, las cuales deben ser superadas mediante propuestas estèticas emergentes como el uso de imaginarios, plataformas virtuales, redes para diversas comproducciones.

Respecto del uso de las plataformas virtuales convergemos con la investigación desarrollada con Cerrón \& Rojas, (2018) de lo cual se colige una relación análoga en la concepción de la comproducción virtual de ecofábulas como proceso de la visibilización interconectiva de los conocimientos considerados en el texto imbricados como los del lector con el uso de las aulas virtuales como www.padlet.com.

\section{Conclusión}

El nivel de eficacia de imaginario estético en la comproducción virtual de ecofábulas es alto en los estudiantes de la Facultad de Educación de la Universidad Nacional del Centro del Perú, porque las sensibilidades sociales del conocimiento, filosofía, educación, política e ideología; la interacción simbólica de la imagen de lo típico individual, racional emocional, objetivo subjetivo, así como lo bello feo, trágico cómico, heroico, vil, sublime, humano frívolo, sutil grotesco facultan la comproducción virtual de la situación inicial, inicio del conflicto, solución del conflicto, situación estable de la ecofábula emergente, como lo evidencia la publicación de ecofábulas comproducidas en https://padlet.com/josecin4/ecofavirtual y la estimación del p-valor: $0.00=0 \%$ con un nivel de significancia de $\alpha=0,05$. 


\section{Referencias bibliográficas}

Ariste, Estela \& Zapana, S. (2011) La ecofábula como estrategia para el desarrollo de la habilidad comunicativa corporal en la institución educativa $N^{\circ} 30218$ - Madre Teresa de Calcuta-El Tambo: Universidad Nacional del Centro del Perú

Cerrón Rojas, W., \& Rojas López, B. (2015). Habilidades investigativas y formación de valores ecopedagógicos en estudiantes de Educación de la Universidad Nacional del Cenro del Perú . Huancayo: UNCP.

Cerrón Rojas, W., \& Rojas López, B. (2016). Habilidades investigativas y formación de valores ecopedagógicos en estudiantes de Educación de la Universidad Nacional del Cenro del Perú. Huancayo: UNCP.

Cerrón Rojas, Waldemar ; Rojas López, Bertha. (2016). Aulas virtuales y habilidades investigativas en estudiantes de Educación de la Universidad Nacional del Centro del Perú. Huancayo - Perú: UNCP.

Cerrón y López . (2015). Habilidades investigativas y formación de valores pedagógicos ambientales. Huancayo : UNCP.

Hernández Moredo, L. (2020). El devenir de las ideas estéticas en el siglo XIX cubano. Boletín de Estética, 50, 57-92. https://doi.org/10.36446/be.2020.50.111

Montes de Oca, H. \& Moscoso, E. (2015). Las ecofábulas en la resolución de problemas matemáticos en estudiantes de segundo grado. Lima: Repositorio de la Universidad San Ignacio de Loyola. http://repositorio.usil.edu.pe/handle/USIL/2204

Maccioni, F. (2016). On the threshold of the amphibious voices: the acuatic imaginary in contemporary argentinian poetry. Anclajes, 20(2), 33-50. https://doi.org/10.19137/ anclajes-2016-2023

Montoya, D. (2019). Restauración de redes ecológicas: Escalas espacial y temporal, estabilidad y cambio global. Ecosistemas, 28(2), 11-19. https://doi.org/10.7818/ECOS.1706 ¿A imaginação romântica como neurose? O debate freudiano sobre o animismo e a questão do olhar em E. T. A.

Hoffmann. Pandaemonium Germanicum, 22(38), 122-141. https://doi.org/10.11606/198288372238122

Neuburger, A. (2020). El presente y sus restos. Arte, literatura e imagen en la estética contemporánea. LA PALABRA, 37, 41-56. https://doi.org/10.19053/01218530.n37.2020.8950

Pavón, R. I. (2016, 28 abril). Una reflexión para la comprensión de los imaginarios sociales. http:// www.scielo.org.pe/scielo.php?script=sci_arttext\&pid=S2219-71682016000100006

(C) Los autores. Este artículo es publicado por la Horizonte de la Ciencia de la Unidad de Posgrado de la Facultad de Educación de la Universidad Nacional del Centro del Perú. Este es un artículo de acceso abierto, distribuido bajo los términos de la Licencia Atribución-No Comercial 4.0 Internacional.(https://creativecommons.org/licenses/by-nc/4.o/), que permite el uso no comercial y distribución en cualquier medio, siempre que la obra original sea debidamente citada. 